\title{
Towards the Estimation of an Efficient Benchmark Portfolio: The Case of Croatian Emerging Market
}

\author{
Denis Dolinar* \\ Davor Zoričić* \\ Antonija Kožul
}

\begin{abstract}
The fact that cap-weighted indices provide an inefficient risk-return trade-off is well known today. Various research approaches evolved suggesting alternative to cap-weighting in an effort to come up with a more efficient market index benchmark. In this paper we aim to use such an approach and focus on the Croatian capital market. We apply statistical shrinkage method suggested by Ledoit and Wolf (2004) to estimate the covariance matrix and follow the work of Amenc et al. (2011) to obtain estimates of expected returns that rely on risk-return trade-off. Empirical findings for the proposed portfolio optimization include out-of-sample and robustness testing. This way we compare the performance of the capital-weighted benchmark to the alternative and ensure that consistency is achieved in different volatility environments. Research findings do not seem to support relevant research results for the developed markets but rather complement earlier research (Zoričić et al., 2014).
\end{abstract}

Keywords: efficient benchmark, cap-weighted indices, risk-return trade-off, undeveloped and illiquid markets

JEL Classification: G11, G12

\section{Introduction}

In practice of portfolio management the investment professionals relying on the capital asset pricing model (CAPM) have long used the market capitalisation weighted indices as a proxy for the optimal portfolio of risky assets following Markowitz

* Denis Dolinar and Davor Zoričić are at University of Zagreb, Faculty of Economics \& Business, Zagreb, Croatia.

** Antonija Kožul is at McKinsey \& Company - Global management consulting, Zagreb, Croatia. 
(1959) and Sharpe $(1963 ; 1964)$. However, the work of Haugen and Baker (1991) and Grinold (1992) and the research that followed inevitably led to a conclusion that the cap-weighted indices provide inefficient risk-return trade-off. This implies that if they are used as a proxy for the CAPM's optimal portfolio of risky assets there will be no adequate compensation for the systematic risk.

Encouraged by the growing body of literature this paper tests the possibility of introducing an efficient market index benchmark in an emerging market environment such as the Croatian one. A comprehensive overview of more efficient benchmarks that could each serve as an alternative to the market cap-weighted index can be found in Amenc, Goltz and Martellini (2013), but in this research the approach taken by Amenc, Goltz, Martellini and Retkowsky (2011) regarding the estimation of the MSR portfolio is followed, which includes out-of-sample and robustness testing. The approach requires that stocks' returns and the covariance matrix be estimated as input parameters. Based on the research undertaken by Martellini (2008), Amenc et al. (2011) introduced semi-deviation of stocks' returns as a proxy for their return, which should improve parameter estimation especially in the Croatian market environment. When it comes to covariance matrix estimation statistical shrinkage method is used as proposed by Ledoit and Wolf (2004).

Earlier research by Zoričić, Dolinar and Kožul (2014) explored the same problem but only included testing the efficiency of proposed benchmarks ex post. Surprisingly it is hard to find similar research for other emerging markets, although it is relatively easy to find announcements of investment funds and index providers indicating that they have already launched or will soon be launching smart beta indices or ETF's for a certain market or a region.

\section{Methodology}

In an overview by Amenc et al. (2013) 11 different efficient benchmarks strategies are presented. Explaining all of them is beyond the scope of this research since the focus of this paper is the Maximum Sharpe Ratio (MSR) portfolio estimation. Estimation of the MSR portfolio is pursued because such benchmark portfolio is optimal by construction. For all other proposed benchmarks assumptions have to be met in order to claim that those portfolios can be attributed maximum Sharpe ratio. If for instance Global Minimum Variance (GMV), Maximum Decorrelation (MDC) and Equally Weighted (EW) benchmarks are more closely examined for all of them all stocks would have to bear the same expected return $\left(\mu_{i}=\mu\right)$. In addition for MDC volatility of all stocks would have to be equal $\left(\sigma_{i}=\sigma\right)$ and for EW volatility and correlations of all stocks would have to be equal $\left(\sigma_{i}=\sigma, \rho_{i j}=\rho\right)$ (Amenc et al., 2013, p. 10-14). Mentioned strategies are presented below in Table 1. 
Table 1: Comparison of Maximum Sharpe Ratio and other selected efficient strategies

\begin{tabular}{|c|c|c|c|}
\hline Strategy & Weighting scheme & $\begin{array}{l}\text { Required } \\
\text { parameter }\end{array}$ & $\begin{array}{l}\text { Optimality } \\
\text { conditions }\end{array}$ \\
\hline Maximum Sharpe Ratio (MSR) & $w=\frac{\Sigma^{-1} \mu}{1^{\prime} \Sigma^{-1} \mu}$ & $\mu_{i}, \sigma_{i}, \rho_{i j}$ & $\begin{array}{l}\text { Optimal by } \\
\text { construction }\end{array}$ \\
\hline Max Deconcentration / Equal Weights & $\boldsymbol{w}=\frac{1}{N} \mathbf{1}$ & None & $\begin{array}{l}\mu_{i}=\mu \\
\sigma_{i}=\sigma \\
\rho_{i j}=\rho\end{array}$ \\
\hline Global Minimum Variance (GMV) & $w=\frac{\Sigma^{-1} 1}{1^{\prime} \Sigma^{-1} 1}$ & $\sigma_{i}, \rho_{i j}$ & $\rho_{i j}=\mu$ \\
\hline Max Decorrelation (MDC) & $w=\frac{\Omega^{-1} 1}{1^{\prime} \Omega^{-1} 1}$ & $\rho_{i j}$ & $\begin{array}{l}\mu_{i}=\mu \\
\sigma_{i}=\sigma\end{array}$ \\
\hline
\end{tabular}

Source: Amenc, N., Goltz, F. \& Martellini, L. (2013). Smart Beta 2.0. Nice, France: EDHEC-Risk Institute

It is hard to expect that any, let alone all, of such assumptions will ever be met in practice but on the other hand it has to be noted that reduction in optimality is compensated by reduced estimation risk as fewer parameters need to be estimated in the case of the GMV and DMC portfolios relative to the MSR portfolio. However, the GMV benchmark has a clear built-in bias towards low-volatility stocks which can lead to overexposure to low volatility industry sectors. MDC benchmark does not concentrate portfolio in low volatility stocks but this comes at the cost of the assumption that volatilities are equal across all stocks (Amenc et al., 2013, p. 7, p. 32).

The risk related to the MSR portfolio estimation is that it requires estimation of more parameters (the expected returns, volatilities and correlations of stocks under consideration) than any other strategy with estimation of expected return, presenting the biggest challenge as reported in Amenc et al. (2013, p. 11). However, as demonstrated in Martellini (2008) total volatility of a stock can be used as a proxy for the stock's expected excess return (expected return in excess of the risk-free rate). Based on this finding Amenc et al. (2011) adopt such an approach but also take it a step further by taking into account higher-order moments, i.e. they introduced a downside risk measure by calculating the semi-deviation of stock returns. In this way only the deviations below the mean are measured which is what the investors should really be concerned about. Thus, the higher the risk measured in this way the higher the investors' expected return should be. In the end in order to estimate the expected returns Amenc et al. (2011) follow sorting approach by Fama and French (1992). Semi-deviations are calculated using the following formula:

$$
\mu_{i}=\sqrt{\frac{\sum_{t=1}^{T}\left\{\min \left[\left(r_{i, t}-\bar{r}_{i}\right), 0\right]\right\}^{2}}{T}}
$$


where $\mu_{i}$ is the semi-deviation of realized returns of stock $i, r_{i, t}$ is the realized return of stock $i$ in period $t, \bar{r}_{i}$ is the arithmetic average of the realized returns, and $T$ represent number of periods in the sample. In this research expected returns are then estimated by forming quartile portfolios for which the semi-deviations' median is calculated. Each stock in a quartile portfolio is attributed the median of the quartile it belongs to as a proxy of its excess expected return.

When it comes to estimating the stocks' covariance matrix, approach proposed by Ledoit and Wolf (2004) is applied in order to reduce the estimation error contained in the sample covariance matrix. The authors propose a transformation (referred to as shrinkage) that tends to pull the most extreme coefficients towards more central values. It is described by the following formula:

$$
\boldsymbol{\Sigma}=\delta \boldsymbol{F}+(1-\delta) \boldsymbol{S}
$$

where $\Sigma$ is the estimation of true covariance matrix of expected returns, $S$ is the sample covariance matrix of realized returns and $\boldsymbol{F}$ is a highly structured covariance matrix estimator (referred to as the shrinkage target). Ledoit and Wolf (2004) suggest a simplification for the matrix $\mathrm{F}$ by adopting the constant correlation model which means that the average of all the sample correlations is the estimator of the common constant correlation. Thus matrix $\mathrm{F}$ represents the sample constant-correlation covariance matrix of realized returns. Parameter $\delta$ is the shrinkage constant which minimizes the expected value of loss in the process of estimation of true covariance matrix. Basically, $\delta$ is a number between 0 and 1, whose estimation requires the calculation of additional estimators defined by the following formulas:

$$
\begin{gathered}
\hat{\delta}=\max \left\{0, \min \left\{\frac{\hat{\kappa}}{T}, 1\right\}\right\} \\
\hat{\kappa}=\frac{\hat{\pi}-\hat{\rho}}{\hat{\gamma}} \\
\hat{\pi}=\sum_{i=1}^{N} \sum_{j=1}^{N}\left\{\frac{1}{T} \sum_{t=1}^{T}\left[\left(r_{i, t}-\bar{r}_{i}\right)\left(r_{j, t}-\bar{r}_{j}\right)-s_{i j}\right]^{2}\right\} \\
\hat{\rho}=\sum_{i=1}^{N} \hat{\pi}_{i i}+\sum_{i=1}^{N} \sum_{j=1, j \neq i}^{N}\left\{\frac{\bar{u}}{2}\left(\sqrt{\frac{s_{j j}}{s_{i i}}} \hat{\vartheta}_{i i, i j}+\sqrt{\frac{s_{i i}}{s_{j j}}} \hat{\vartheta}_{j j, i j}\right)\right\} \\
\left.\hat{\gamma}=\sum_{i=1}^{N} \sum_{j=1}^{N}\left(f_{i j}-s_{i j}\right)^{2}\right) \\
\hat{\vartheta}_{i i, i j}=\frac{1}{T} \sum_{t=1}^{T}\left\{\left[\left(r_{i, t}-\bar{r}_{i}\right)^{2}-s_{i i}\right]\left[\left(r_{i, t}-\bar{r}_{i}\right)\left(r_{j, t}-\bar{r}_{j}\right)-s_{i j}\right]\right\}
\end{gathered}
$$


Here, $s_{i j}$ represent values form the sample covariance matrix $S, f_{i j}$ represents values from the sample constant-correlation covariance matrix $\boldsymbol{F}$, and $\bar{u}$ is the average correlation coefficient of stock returns in the sample.

Based on estimated expected returns and covariance matrix the MSR portfolio's weights are calculated by solving the following formula:

$$
\boldsymbol{w}^{*}=\arg \max _{\boldsymbol{w}} \frac{\boldsymbol{w}^{\prime} \boldsymbol{\mu}}{\sqrt{\boldsymbol{w}^{\prime} \boldsymbol{\Sigma} \boldsymbol{w}}}
$$

Here $w^{*}$ are the efficient weights (i.e. the vector of weights) that will be used in the efficient index, $\mu$ is the vector of expected returns in excess of the risk-free rate, and $\Sigma$ is the covariance matrix for expected returns of these constituents. In the process of optimization constraints (minimum, maximum and total) are imposed by the following formulas:

$$
\begin{gathered}
\frac{1}{\lambda N} \leq w_{i}^{*} \leq \frac{\lambda}{N} \\
\sum_{i=1}^{N} w_{i}^{*}=1
\end{gathered}
$$

where $w_{i}^{*}$ is the efficient weight of stock $i, N$ is the number of stocks in the CROBEX index at the time, and $\lambda$ is a flexibility parameter. Such constraints do not allow short selling. These constraints, together with the turnover limitation rules, are imposed following the work of Amenc et al. (2011).

Finally, the MSR portfolio is estimated for multiple data samples based on the formulas above and is tested out-of-sample every time following the work of Amenc et al. (2011). Rolling window (moving over the entire observed period) is used to sample the data. For each sample the performance of the estimated MSR portfolio ${ }^{1}$ is compared out-of-sample to the performance of the market cap-weighted counterpart. This is explained in more detail in the next section.

\section{Data}

This paper focuses on the stocks that were listed on the Zagreb Stock Exchange (ZSE) and included in the CROBEX index in the period from March 2005 till March 2016. As can be seen from the Figure 1 below the market experienced a significant rise and sharp decline before levelling out at market capitalisation of around 130 billion kuna on average in the last 8 years. Changes in the market capitalisation have been accompanied by changes in liquidity as presented by the turnover ratio which has remained well below $3 \%$ in the last 5 years. 
Figure 1: Market capitalisation (in millions of kunas) and turnover ratio on ZSE (2005-2016)

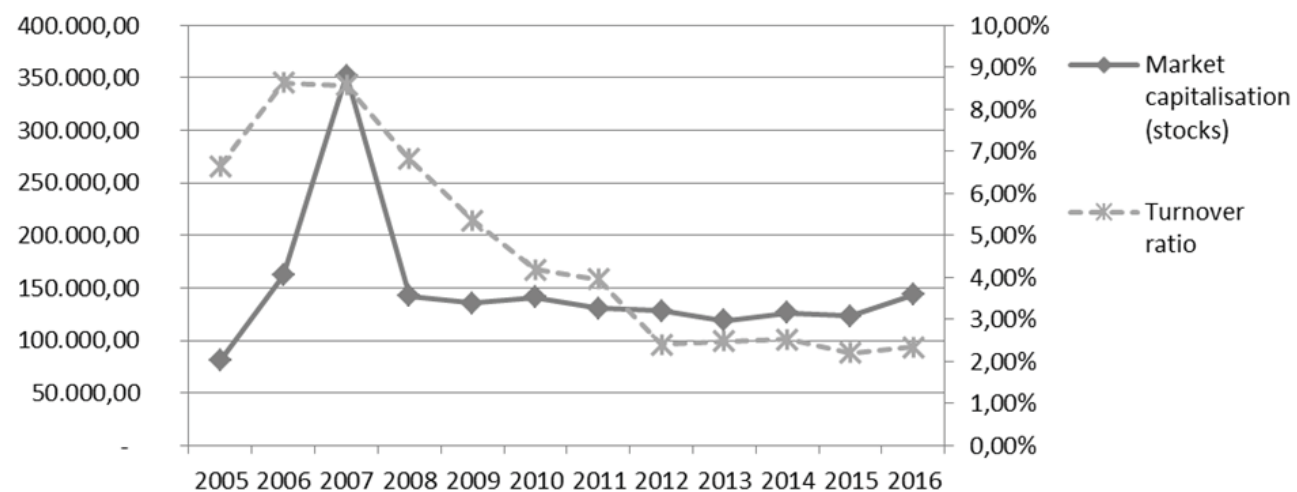

Source: The Zagreb Stock Exchange

The years of stagnation in market capitalisation and falling liquidity depicted in Figure 1 characterise this market as undeveloped and illiquid. It can be noted that if, for instance, EDHEC Risk Institute's Scientific Beta Universe Construction Rules (2016, December) for inclusion in the Emerging Market Universe were applied to the above data Croatian market would just fall short of the size criterion (minimum of 20 billion USD market capitalisation on average over the last 3 years). In terms of meeting the liquidity criterion (minimum 5\% over the last 3 years) Croatian market is much further away. However, if only the period of high volatility in the market was analysed (2005-2009) both criteria would meet.

The research is based on the CROBEX index since it is the oldest and largest stock index in the Croatian capital market. Introduced in 1997 by the ZSE, it is a market cap-weighted broad-based index designed to reflect the movement of the entire market. Also it is a price index meaning that the dividends are not accounted for its calculation. In the observed period the CROBEX index composition varied from 17 to 32 stocks, with the average being close to 25 stocks. A total number of 61 stocks were included in the index over the entire analysed period and all of them were included in the analysis as the composition of the proposed efficient benchmark is matched to the CROBEX for the purpose of performance comparison.

The estimation process is based on monthly total returns which include both capital gains and dividend yields (where applicable). Out-of-sample performance testing is based on semi-annual returns for which dividend yield is ignored, since the CROBEX index is a price index (it doesn't take into account dividend yield and is revised semi-annually). It should also be noted that the numerator in the Sharpe ratio which is being maximized in the MSR approach represents the return in excess of the risk-free rate. All the estimations are therefore actually conducted based on the expected excess return for each stock. The yield on the Ministry of Finance three- 
month treasury-bill at the moment of issuing denominated in local currency is used as a proxy for the risk-free rate.

Since the CROBEX index is revised regularly on semi-annual basis (in March and September) in order to match the actual constitution of the CROBEX as closely as possible, the MSR portfolio estimation is always performed based on the updated index composition. This also implies that rebalancing is done every 6 months (irregular revisions of the CROBEX index are ignored). Thus, during the 11-year-observation-period, the MSR portfolio has been estimated (i.e. rebalanced) 22 times over. At every rebalancing sample data containing monthly returns for the last 3 years (36 observations for each stock) is used in the estimation process. The rebalancing is done after the close of the third Friday of March and September (i.e. rebalancing corresponds with the dates of regular revisions of the CROBEX index).

Based on the above data sources for the research include the ZSE, the Central Depository \& Clearing Company and the Ministry of Finance of the Republic of Croatia.

\section{Research Findings}

The analysis is carried out by estimating the described parameters for each data sample (22 data samples each containing monthly returns for the last 3 years). The resulting estimated optimal weights of the MSR portfolio are then applied out-ofsample for each one of them (6-months ahead) to test its performance. Overall out-ofsample performance (for all data samples) is compared to the market-cap counterpart (CROBEX). We used Sharpe ratio as a key performance measure. Average returns reported refer to geometric average and were calculated based on semi-annual returns. Volatility refers to the standard deviation of the semi-annual returns. In order to test the robustness of the obtained results entire observation period is divided in two sub-periods (high and low volatility period) for which the results are reported separately. The results are presented in the table below.

Table 2: Out-of-sample performance and robustness of the estimated benchmark

\begin{tabular}{|c|l|r|r|r|r|}
\hline & & CROBEX & MSR $(\lambda=4)$ & MSR $(\lambda=2)$ & EW \\
\hline \multirow{2}{*}{$\begin{array}{c}\text { Entire observation period } \\
\text { March 2005 - March 2016) }\end{array}$} & Average return & $-0.82 \%$ & $-3.13 \%$ & $-3.49 \%$ & $-3.74 \%$ \\
\cline { 2 - 6 } & Volatility & $24.06 \%$ & $22.20 \%$ & $25.04 \%$ & $26.02 \%$ \\
\cline { 2 - 6 } & Sharpe ratio & -0.034 & -0.141 & -0.140 & -0.144 \\
\hline \multirow{2}{*}{$\begin{array}{c}\text { High volatility period } \\
(\text { March 2005 - September 2009) }\end{array}$} & Average return & $1.43 \%$ & $0.18 \%$ & $-0.50 \%$ & $-0.69 \%$ \\
\cline { 2 - 6 } & Volatility & $35.96 \%$ & $32.99 \%$ & $37.76 \%$ & $39.32 \%$ \\
\cline { 2 - 6 } & Sharpe ratio & 0.040 & 0.005 & -0.013 & -0.017 \\
\hline \multirow{2}{*}{$\begin{array}{c}\text { Low volatility period } \\
\text { September 2009-March 2016) }\end{array}$} & Average return & $-2.35 \%$ & $-5.35 \%$ & $-5.51 \%$ & $-5.80 \%$ \\
\cline { 2 - 6 } & Volatility & $10.42 \%$ & $9.31 \%$ & $9.13 \%$ & $8.98 \%$ \\
\cline { 2 - 6 } & Sharpe ratio & -0.225 & -0.575 & -0.604 & -0.646 \\
\hline
\end{tabular}

Source: Authors' calculations 
The Table 2 results expose a surprising and dramatic failure of the MSR benchmark out-of-sample estimation for the analysed data. The comparison of results for the MSR portfolio when $\lambda=4$ and $\lambda=2$ reveals that when higher concentration is allowed (i.e. when the value of $\lambda$ is higher) the optimizer will exploit the opportunity to concentrate more in certain stock(s) and thus improve the benchmark's performance. Equally-weighted (EW) portfolio (i.e. maximum deconcentration portfolio) performance is added as an additional proof of the identified pattern. When all presented results in the table are considered it is obvious that deconcentration of portfolio erodes its performance as the MSR portfolio with $\lambda=4$ ranks second and the EW portfolio ranks fourth (last) for all three segments specified. Better performance by CROBEX comes as a surprise not only because it outperforms MSR portfolio overall but because it does so even during the worst period of financial crisis (it bears lower losses). Also, this is in contrast to what was expected because the results of the earlier research by Zoričić et al. (2014) seemed to suggest that EW performs better than cap-weighted counterpart (based on CROBEX). But the results of the research were based on the "ex post" analysis and this is the first time to the authors' best knowledge that out-of-sample analysis was performed for an illiquid and undeveloped emerging market.

However surprising the above results may be there's more than a few factors that have to be considered as possible contributors to such outcome. First of all, it is clear that even in the case of the most developed financial markets any deviation from the market cap-weighing leads to higher exposure to less liquid stocks. Although, the results presented for the developed markets proved robust regarding the liquidity of the stocks, the results here likely support the opposite (although additional testing is required). ${ }^{2}$ Also it has to be noted that the MSR but also the GMV and the MDC portfolios all have implicit small cap exposure. Just as in the case of exposure to less liquid stocks, the exposure to small capitalization stocks still improves the diversification effect ${ }^{3}$ relative to the cap-weighted index in the developed markets (Amenc et al., 2013, p. 7, p. 17-19). In the case of the undeveloped financial market again it seems that exposure to small cap can prove to be especially costly. As already pointed out by Zoričić et al. (2014) the deconcentration of the portfolio does not necessarily result in the desired stronger diversification effect as some of the large-cap stocks also proved to be among least correlated in the market. Therefore, even in the case of this ex-post (in-sample) based analysis the pure market-cap weighted portfolio proved to be the most efficient one. ${ }^{4}$ The EW portfolio proved to be the worst both in the in-sample and out-of-sample testing as it implicitly exposes an investor to both small cap and liquidity risks by naively maximizing the deconcentration effect.

Some other peculiarities related to the illiquid and undeveloped also have to be taken into account. The market liquidity is generally low, which is in itself problematic, but in extreme situations the trading activity regarding some stocks may be so low that there is no continuous trading during the course of one month regardless of 
their inclusion in the CROBEX. The return calculated for that stock does not necessarily always correspond to a period of one month and it is hardly reliable. Most importantly in the CROBEX the weights of these stocks may be small and therefore such is the impact. On the other hand, for the MSR portfolio estimation and out-ofsample testing they may be much more significant. Apart from this on some occasions there is missing data, even in the case of the biggest companies in the market. Croatian Telekom presents such a special case as the company went public and was immediately included in CROBEX (with a significant weight) but with no trading history available at that point in time. Lastly, it was expected that using the semi-deviation of stocks' return as a proxy for the stocks' estimated return would improve these estimates. Although the theoretical background (and empirical in the case of developed markets) for this is solid it has to be noted that analysis such as in Martellini (2008) should be conducted in order to verify the hypothesis in the emerging market environment.

In the end the question remains if an efficient benchmark for the illiquid and undeveloped markets can be estimated. Especially regarding the last problematic issue mentioned it should be pointed out that an efficient benchmark portfolio other than the MSR might prove to outperform the cap-weighted index in such environment. Although the MSR portfolio is optimal by construction and represents the true efficient portfolio in theory the estimation risks one faces when using such a portfolio as a benchmark may exceed the benefit of bearing no optimality risk. In fact it has been shown that when estimation risk is included the GMV portfolio dominates the MSR portfolio. Simply put the estimation of the optimal portfolio comes at a high cost with the estimation of expected return parameter being critical (Amenc et al., 2013, p. 10-13, p. 22).

Based on all these findings in an environment plagued with unreliable and missing data the best approach may very well prove to be to focus on one thing - risk reduction. On the one hand it does seem odd to focus on the minimum risk portfolio in a risky environment (high reward should accompany high risk), but on the other it makes sense if it is hard to estimate true risk and reward due to tarnished data and if due to specific circumstances there seems to be relatively few desirable diversification opportunities. Also for some data samples in this research low correlation between stocks' return and volatility was identified which is sometimes viewed as an argument in favour of the GMV portfolio as a benchmark (Amenc et al., 2013, p. 15).

However, it should be stressed here that the GMV portfolio can lead to overexposure to low volatility stocks (i.e. sectors such as utilities) (Amenc et al., 2013, p. 7-9, p. 19). In order to avoid this and to take everything above into the account future research should use constrained optimization techniques that define maximum or minimum exposure to sectors and other thresholds regarding stock selection criteria (for instance based on liquidity and market capitalization). Also, other methods for the covariance matrix estimation, such as principal component analysis should be tested. 


\section{Conclusion}

Over the last 20 years the research showed that the market-cap weighted indices provide an inefficient risk-return trade-off and alternative, more efficient, benchmarks were proposed as a substitute for the purpose of portfolio management. The success of the efficient benchmarks in the developed markets is well documented especially regarding the most promising and sophisticated strategies: the MSR, GMV and MDC portfolios to name a few.

The results of this research show that surprisingly in the case of an illiquid and undeveloped financial market such as the Croatian one the market-cap index (CROBEX) by far outperforms the estimated MSR benchmark portfolio in an outof-sample testing over the entire observed period regardless of market conditions (expansion or recession). The main reasons of failure point to exposure to less liquid and small cap stocks as deviation from market cap-weighing is applied. In fact the research results show that results tend to get worse with stronger deconcentration. Thus if the MSR portfolio is estimated by imposing stronger constraints (on its weights) regarding deconcentration it performs worse than the same portfolio with weaker constraints allowing the weights to be much more similar to the ones in the cap-weighted benchmark. Moreover, if deconcentration is set to maximum following the naïve equally-weighted approach the results are the worst of all.

As much as good it is to find that at least the naïve strategy performs the worst still the question remains if there is a way to provide a more efficient benchmark than the market cap-weighted index for the illiquid and undeveloped emerging markets. Based on the findings of empirical research conducted for the developed markets it seems that especially in the case of illiquid and undeveloped markets the cost of optimality in the MSR portfolio estimation is too high. Namely, the MSR is the optimal benchmark portfolio according to theory but optimality does not seem to compensate nearly enough for high estimation risks involved. Therefore, it seems that future research should test the GMV portfolio as a benchmark in the illiquid and undeveloped market as it has shown the potential to dominate the MSR portfolio even in the developed markets. Since the estimation of the GMV portfolio relies solely on the estimation of the covariance matrix an effort should be made to further improve upon this especially regarding missing and unreliable sample data.

\section{ENDNOTES}

${ }^{1}$ Following Amenc et al. (2011), if the change in estimated portfolio weights is less than $50 \%$ of port-
folio value estimates based on the previous data sample are used. They are only adjusted for the change
in the CROBEX composition for the analysed period of time. Exiting constituents are removed and 
new entries are included with the minimum weight (based on the equation 10). All weights are then scaled to meet the requirement that they have to sum to $100 \%$.

${ }^{2}$ However it should be pointed out that in the period $2005-2012$ on average $77.6 \%$ of the trading volume and around $50 \%$ of total market capitalization on the ZSE was related to the stocks included in the CROBEX index. Furthermore the top 5 cap-weighted stocks included in the CROBEX were on average attributed with $62.1 \%$ of the trading volume and on average accounted for $75.2 \%$ of the market capitalization of all stocks included in the CROBEX in the same period.

${ }^{3}$ Although the effect for the MSR and the DMC portfolio is the strongest for the small cap segment and the weakest in the large cap segment. For the GMV the weakest effect was reported in the small cap segment.

${ }^{4}$ In the research the market-cap weighted portfolio with no constraints outperformed market-cap weighted portfolio with maximum weight constrained to 10\% and CROBEX (free float adjusted and max. weight constrained to $10 \%$ ). In fact CROBEX as the most deconcentrated portfolio proved to be the worst of three. The only worse performance came from the equally-weighted portfolio.

\section{REFERENCES}

Amenc, N., Goltz, F. \& Martellini, L. (2013). Smart Beta 2.0. Nice, France: EDHEC-Risk Institute. Amenc, N., Goltz, F., Martellini, L. \& Retkowsky, P. (2011). Efficient Indexation: An Alternative to Cap-Weighted Indices. The Journal of Investment Management. 9(4), 1-23.

Fama, E. F. \& French, K. R. (1992). The cross-section of expected stock returns. The Journal of Finance. 47(2), 427-465. DOI: 10.1111/j.1540-6261.1992.tb04398.x.

Grinold, R. C. (1992). Are Benchmark Portfolios Efficient? The Journal of Portfolio Management. 19(1), 34-40. DOI: 10.3905/jpm.1992.34.

Haugen, R. A. \& Baker, N. L. (1991). The Efficient Market Inefficiency of Capitalization-Weighted Stock Portfolios. The Journal of Portfolio Management. 17(3), 35-40. DOI: 10.3905/.jpm.1991.409335.

Ledoit, O. \& Wolf, M. (2004). Honey, I shrunk the sample covariance matrix. The Journal of Portfolio Management. 30(4), 110-119. DOI: 10.3905/jpm.2004.110.

Markowitz, H. M. (1959). Portfolio Selection: Efficient Diversification of Investments. New York, USA: John Wiley \& Sons, Inc.

Martellini, L. (2008). Toward the Design of Better Equity Benchmarks: Rehabilitating the Tangency Portfolio from Modern Portfolio Theory. The Journal of Portfolio Management. 34(4), 34-41. DOI: $10.3905 / \mathrm{jpm} .2008 .709978$.

ScientificBeta. (2016, December). ERI Scientific Beta Universe Construction Rules. Retrieved February 27, 2017, from http://www.scientificbeta.com/download/file/grd-universe

Sharpe, W. F. (1963). A Simplified Model of Portfolio Analysis. Management Science. 9(2), 277-293. DOI: $10.1287 / \mathrm{mnsc} .9 .2 .277$.

Sharpe, W. F. (1964). Capital Asset Prices: A Theory of Market Equilibrium under Conditions of Risk. The Journal of Finance. 19(3), 425-442. DOI: 10.1111/j.1540-6261.1964.tb02865.x.

Zoričić, D., Dolinar, D. \& Kožul, A. (2014). The Market Index Benchmark and Adequate Compensation for Systematic Risk in an Illiquid and Undeveloped Financial Market. In D. Miloš Sprčić (Eds.), Risk management: Strategies for Economic Development and Challenges in the Financial System (pp. 257-277). New York, USA: Nova Science Publishers, Inc. 